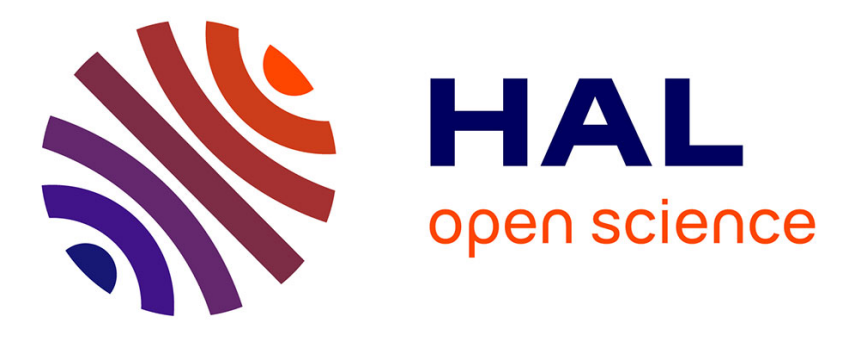

\title{
Experimental flights of adaptive patterns for cloud exploration with UAVs
}

\author{
Titouan Verdu, Nicolas Maury, Pierre Narvor, Florian Seguin, Gregory \\ Roberts, Fleur Couvreux, Grégoire Cayez, Murat Bronz, Gautier \\ Hattenberger, Simon Lacroix
}

\section{To cite this version:}

Titouan Verdu, Nicolas Maury, Pierre Narvor, Florian Seguin, Gregory Roberts, et al.. Experimental flights of adaptive patterns for cloud exploration with UAVs. IROS 2020, IEEE/RSJ International Conference on Intelligent Robots and System, Oct 2020, Las Vegas (on line), United States. pp 1429-1435, 10.1109/iros45743.2020.9341408 . hal-02994385

\section{HAL Id: hal-02994385 \\ https://hal.laas.fr/hal-02994385}

Submitted on 7 Nov 2020

HAL is a multi-disciplinary open access archive for the deposit and dissemination of scientific research documents, whether they are published or not. The documents may come from teaching and research institutions in France or abroad, or from public or private research centers.
L'archive ouverte pluridisciplinaire HAL, est destinée au dépôt et à la diffusion de documents scientifiques de niveau recherche, publiés ou non, émanant des établissements d'enseignement et de recherche français ou étrangers, des laboratoires publics ou privés. 


\title{
Experimental flights of adaptive patterns for cloud exploration with UAVs
}

\author{
Titouan Verdu ${ }^{1,2}$, Nicolas Maury ${ }^{3}$, Pierre Narvor $^{2}$, Florian Seguin ${ }^{2}$, Gregory Roberts ${ }^{3}$, \\ Fleur Couvreux ${ }^{3}$, Grégoire Cayez ${ }^{3}$, Murat Bronz ${ }^{1}$, Gautier Hattenberger ${ }^{1}$, Simon Lacroix ${ }^{2}$
}

\begin{abstract}
This work presents the deployment of UAVs for the exploration of clouds, from the system architecture and simulation tests to a real-flight campaign and trajectory analyzes. Thanks to their small size and low altitude, light UAVs have proven to be adapted for in-situ cloud data collection. The short life time of the clouds and limited endurance of the planes require to focus on the area of maximum interest to gather relevant data. Based on previous work on cloud adaptive sampling, the article focuses on the overall system architecture, the improvements made to the system based on preliminary tests and simulations, and finally the results of a field campaign. The Barbados experimental flight campaign confirmed the capacity of the system to map clouds and to collect relevant data in dynamic environment, and highlighted areas for improvement.
\end{abstract}

\section{INTRODUCTION}

The models developed to understand atmospheric phenomenons are based on the acquisition of data, either remotely with satellites and ground sensors, or in-situ with planes or sounding balloons. In the case of cumulus clouds, which have a small size (about hundreds meters), a rapid evolution and a short lifespan (about half an hour), these sensing methods are not suited and in-situ measurements are missing to properly understand the cloud dynamics [1].

UAVs are well suited to provide in-situ atmospheric data (see e.g. [2], [3]), but sampling cumulus clouds is challenging. No classic geo-referenced flight patterns such as ascending spirals or swathing patterns can be efficiently applied: indeed the cloud location and shape are initially not known, and both evolve over time. Besides, atmosphere scientists are interested by data gathered in specific areas of the clouds, such as its base, its boundaries or its center. Figure 1 illustrates the various areas of interest in a cumulus cloud.

An exhaustive coverage of a cloud using systematic survey patterns is hardly feasible, as it would need to involve dozens of UAVs. The key to gather relevant data is to opt for an adaptive sampling scheme, in which the UAVs servo their flight on the acquired information. These schemes, referred to as "flight patterns", exploit in real-time the data provided by a sensor that measures the liquid water content of the atmosphere through optical extinction measures [4].

Based on prior work on the development of adaptive "flight patterns" [5], this paper presents a new adaptive pattern called "Trinity" suited for small clouds (section IIIB). It also gives an overview of the complete system created

\footnotetext{
${ }^{1}$ ENAC, Université de Toulouse, Toulouse, France

${ }^{2}$ LAAS-CNRS, Université de Toulouse, CNRS, Toulouse, France

${ }^{3}$ METEO-FRANCE Toulouse, Toulouse Cedex 01, France
}

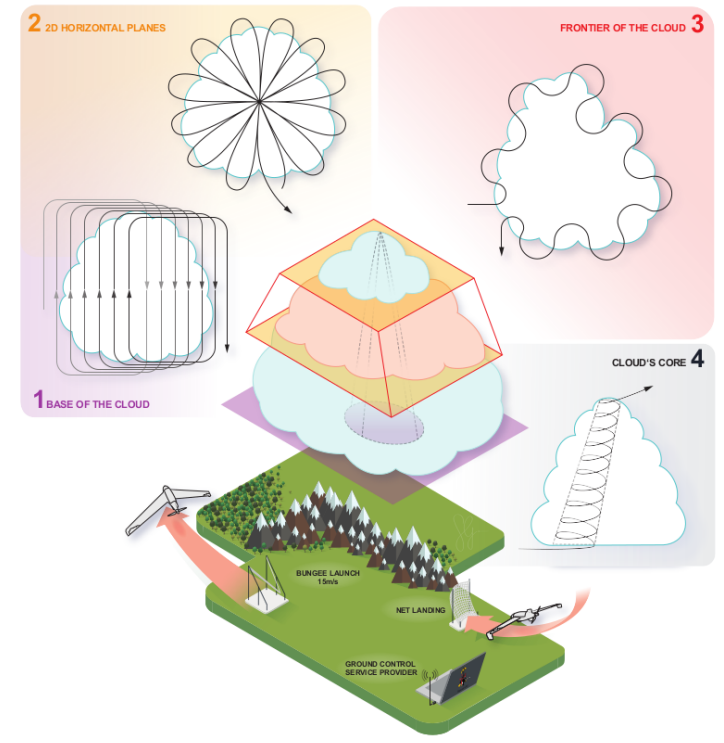

Fig. 1. Cloud interest zones (illustration and design by Sarah Gluschitz)

to sample and map clouds and details the strategy chosen to track it. A validation of this work was done through simulations (section IV) and with real experimental flights that took place during the Barbados flight campaign (section $\mathrm{V})$.

\section{PROBLEM STATEMENT AND APPROACH}

This work is a part of the NEPHELAE project, which is based on the collaboration of atmospheric scientists (Météo France), drone operators and developers (ENAC) and experts in mapping and robotic (LAAS-CNRS), and which follows previous collaborations [6], [7]. NEPHELAE aims to develop and deploy a mapping system composed of a fleet of UAVs to gather data within and around cumulus clouds.

\section{A. Cloud sampling}

The atmospheric scientists use intensive microphysics simulations of the evolution of cumulus clouds to determine factors and parameters which can be representative of the cloud's stage. For instance the radius of the cloud base give a range of values for its height and its vertical flux. These relations between measurements in the cloud (updraft, vertical flux,...) and its geometric parameters (height, radius,...) lead to determine which data have to be gathered :

- The pressure, temperature and humidity (referred as PTU) 
- The wind field and turbulence

- The droplet extinction, which represents how much a droplet reflect the emitted light of a particular sensors equipped with multiple LED (Infrared, Cyan and Orange) and a photo-receptor. This measurement can be use to process the Angström coefficient related to the Liquid Water Content (LWC) [8].

These information give to the atmospheric scientists a clue about the cloud state, from its formation to its dissipation. To maximize the amount of data collected and to obtain an adequate surface coverage of the clouds, several zones of interest are defined, shown in Fig 1, in which the cloud base is represented in purple, the core of the cloud depicted by a conic dotted line, the border of the cloud in red and finally two slices at different altitudes in orange. During the experiments, the sampling focused on the border detection and the data collection on horizontal planes.

The lifespan of the cloud is around $30 \mathrm{~min}$ and according to its actual state the objective was to select the most interesting zone to explore. The frequency of exploration of each part is then adapted according to the cloud evolution. The constraint of flight endurance should also be considered, and is reducing the time allocated to selecting the proper cloud to about 20 to 30 minutes at most.

\section{B. Cloud mapping system software architecture}

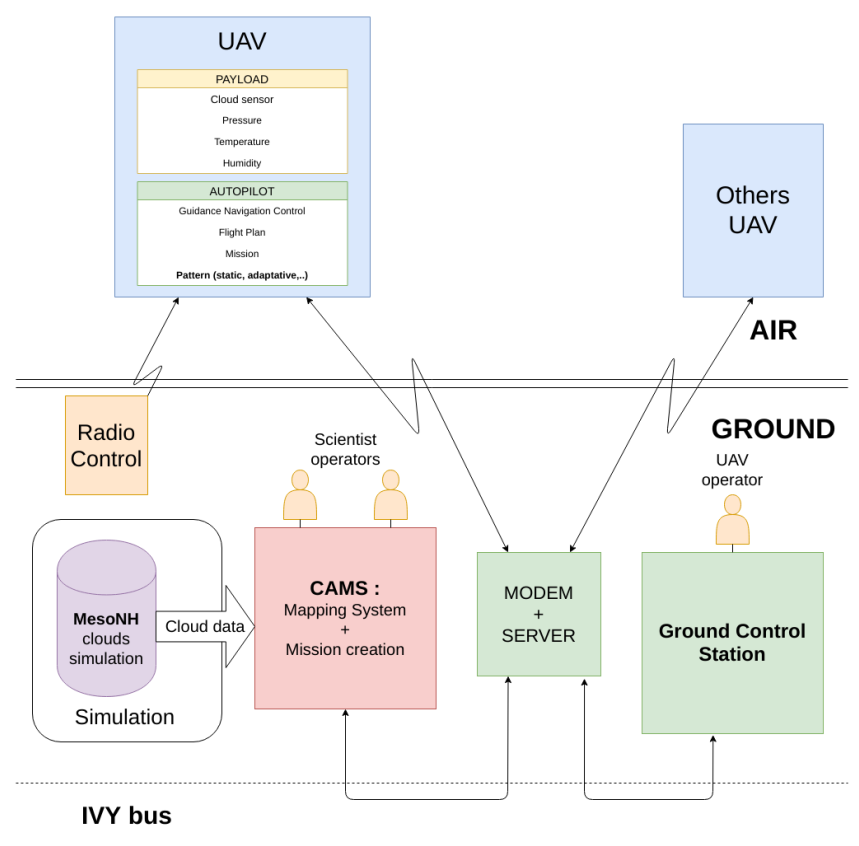

Fig. 2. The global system architecture

The global architecture of the system is presented in Figure 2. The main elements are first the UAV system itself, based on Paparazzi UAS [6] (PPRZ) with the Ground Control Station (GCS) and the on-board Autopilot. Since the built-in flight patterns are not adapted to this project, new flight navigation functions have been added to the system in order to react to on-board sensor measurements.
The payload is connected to the autopilot is providing realtime data to the flight controller and to the ground through the datalink system. Finally the mapping system is running on ground (connected to a cloud database for simulations). Communications between ground agents is based on the PPRZ IVY middleware.

The Cloud Mapping Adaptive System (CAMS, in red) main task is to build the cloud map. It also offers different displays in a web application: an interface for the atmospheric scientist to have a direct view on the sensor value in real time; an interface for the fleet control and finally a main interface to display a map of the current clouds. This map is generated by running a Gaussian Process Regression on the collected data. CAMS also allows to run simulations. To this end, the atmospheric scientists can provide simulations of complex dynamic environments using the MesoNH model (in purple, see section IV for details).

\section{AdAPTIVE FLIGHT PATTERNS}

\section{A. Constraints of geo-referenced patterns}

The set of basic patterns commonly found in autopilot systems are geometric and geo-referenced patterns. For instance, parameters such as the center, radius and vertical speed of a circle pattern can be only changed by hand by the operator. It requires a short response time to modify the flight patterns parameters for an acceptable cloud tracking. This task might be acceptable for a single UAV, but becomes more complex when it comes to control several UAVs of a fleet. Moreover, the targeted cumulus clouds are expending and translating with the horizontal wind. It is therefore necessary to constantly adjust the trajectory of each UAV to stay inside or to repeatedly cross the border. The solution retained is to implement an automatic detection of the cloud border and to let the flight controller adapt the trajectory autonomously.

\section{B. Existing adaptive flight patterns : Rosette and Lace}

The principle behind the development of these flight patterns is based on the detection of the border of a cloud. The Rosette and Lace patterns are described in a previous work [5], only the main points are presented here. The figure 3 illustrates the resulting trajectories of the Rosette (left side) and Lace (right side) patterns.

The rosette pattern is designed to cross the cloud entirely then make a turn around to repeat this task. This pattern offers the possibility to reconstruct many parameters such as cloud dimensions or updraft at the core and the edge of the cloud. However, it can take a lot of time to achieve a complete sampling.

The lace pattern concentrates on the frontier and collects data around it. It appears to be really efficient on cloud that is big enough in comparison with the circle pattern radius. The reason is that every UAVs have roll angle limit that bounds the minimum turning radius. This radius increases with the speed, so a trade-off between the time for a complete turn around the cloud and the sampling step (distance between border crossing) must be found. 

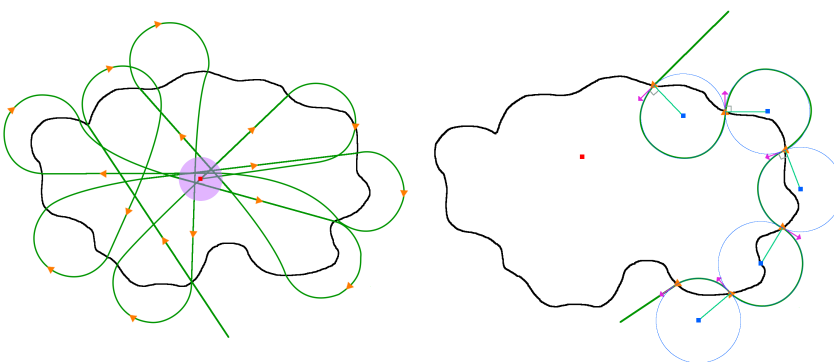

Fig. 3. Rosette (left) and Lace (right) trajectory

These adaptive flight patterns are set with several parameters such as the circle radius, the first turn direction or the vertical speed. This last parameter is important in order to add a third dimension to the sampling. However, the limitation of the cloud sensor's capabilities (measurements disturbed during climbing phase) during real experiments did not allow this parameter to be used during the campaign.

\section{Upgrade on the flight patterns}

The first tests in simulation of these patterns were encouraging but limited by the use of a static cloud. After testing on a new set of simulations with dynamic clouds, some modifications were made to improve the tracking and give more flexibility for the operation during real flights.

The first parameter that was added to the patterns was the possibility to add a drift to the trajectory of the UAV. The estimation of the horizontal wind is provided in real-time by the ground station and the mapping algorithm.

The second parameter that can be changed is the initial side of the circle center and the first rotation direction. The value will be changed automatically according to the strategy of the flight pattern.

The third modification is more specific to the rosette pattern. The initial idea was to use the positions of points detected at the border of the cloud to estimate the center on-board. This point can now be updated directly by the CAMS system that computes a map in real time and uses it to determine the center of the cloud. This solution has proved to be much more efficient as CAMS takes into account the natural drift and has a more sophisticated cloud model.

\section{A new pattern: the Trinity}

The trinity pattern is a new pattern created by the need to sample small clouds. As the strategy of the lace pattern can't be used with clouds that are too small, a variant is proposed.

Figure 4 illustrates the path (in green) of the UAV at different times when executing the Trinity pattern. The red point represent the initial estimated center of the cloud (red point). The first time that the UAV enters, it will process a new circle center (blue point) according to its position (orange triangle) and heading (purple arrow). This first circle center is set on the right side of the UAV and the rotation direction in a clockwise way. Then when it goes out of the cloud, a new circle center is set at its left but the rotation direction is not changed. This action will force the UAV
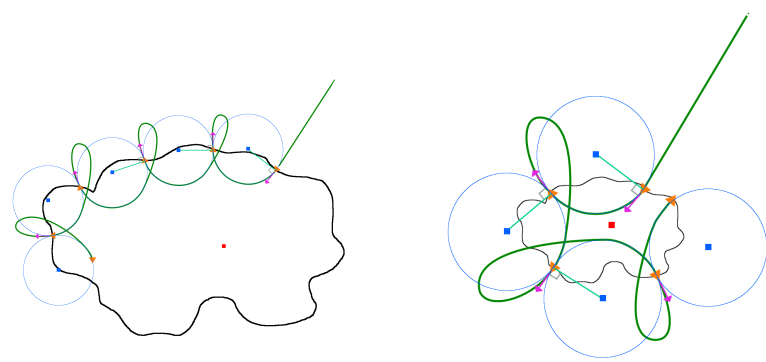

Fig. 4. Trinity trajectory on a big cloud (left side) and on a smaller one (right side)

to adopt a trajectory with sharp turn outside the cloud and smooth turn inside. It is also efficient to follow a cloud during a long time because the period of time outside is reduced.

\section{E. Evaluation metrics}

The question around the evaluation metrics for these adaptive patterns is still under consideration. The objectives of the NEPHELAE project is to collect data in order to build cloud models. From the data point of view, to evaluate this method, the atmospheric scientists have to post process the data collected to see if they can sufficiently rebuild the desired parameters. This part is complex and still an ongoing work. From the adaptive patterns point of view, the most important metrics are the capability of the patterns to adapt themselves to the cloud and never lose track of it. The amount of data collected, the time spent inside a cloud or even the spatial coverage of a pattern may not be fully relevant as evaluation criteria.

\section{Simulations}

A key point of the proposed architecture is the possibility to deploy UAVs in simulated environment. The cloud simulation is based on the MesoNH model [9], which is used to produce a large scale mesh of physical parameters (wind, PTU, LWC) with a spatial resolution of 10 meters and a temporal evolution of 5 seconds for the purpose of this project (representing several $\mathrm{Tb}$ of data after many hours of computation on Meteo-France computers). These data can feed the simulated sensors of the UAVS in order to test the adaptive patterns and CAMS to build the cloud map from the UAV trajectory. The original data set is then used as a reference to compare to map produced in real-time, allowing to adjust the parameters of the regression process. In addition, the PPRZ simulator is based on a flight dynamic simulator called JSBSIM providing realistic flight behavior, including the effect of the wind.

This high-fidelity simulation was a real asset to tune the logic and the parameters of the algorithms, in particular, when dealing with strong wind. The behavior of the patterns and the resulting trajectories are way different between static and dynamic environment, leading to the improvements presented in section III-C.

This architecture also allows to deploy real UAVs in flight while simulating the clouds and payload readings. The main 
goal was to validate the behavior of the system in operational conditions while keeping the UAVs in range of the safety pilot in a reserved airspace. Most components have been qualified for operation this way, except for two points: - the response of the simulated sensor is nearly perfect and does not reflect the actual noise and bias of the cloud sensor; the mismatch between the real wind during flight test and the one from the simulated data produced some unexpected trajectories.

\section{BARBADOS FLIGHT CAMPAIGN AND REAL DEPLOYMENT}

\section{A. Presentation of the campaign and the UAVs}

The NEPHELAE project aims to collect enough in-situ data to model cloud evolution. To this end, a real flight campaign took place in the Barbados island during three weeks. The team took advantage of a bigger atmospheric measurement campaign involving meteorological boats and planes, called EUREC4A [10], to benefit of the special flight authorizations given by the local authorities.

The Barbados is the most eastern island of this part of the Caribbean. Moreover the trade wind coming from the east, is pushing the clouds to develop themselves when they arrive at a few kilometers from the coast. The field for operations was chosen on the east coast facing the ocean and the allocated restricted airspace was a $15 \mathrm{~km}$ by $2.5 \mathrm{~km}$ rectangle with a maximum altitude of 2000 meters.

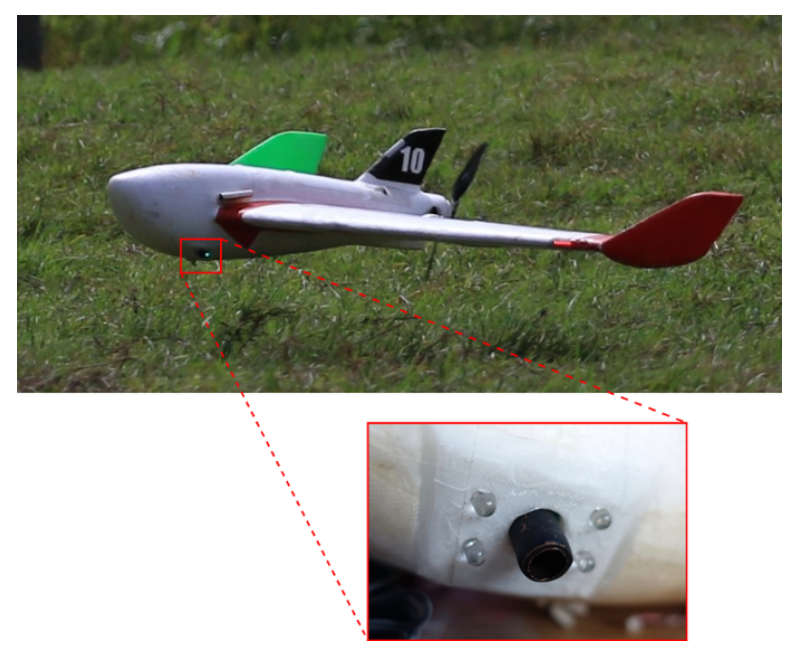

Fig. 5. X6 UAV, close-up on the cloud sensor

The UAVs deployed are foam planes (Skywalker X6, see Figure 5) with a wingspan of $1.5 \mathrm{~m}$, a cruise speed around $16-20 \mathrm{~m} / \mathrm{s}$, a maximum autonomy of one hour and a take-off weight of $2 \mathrm{~kg}$. The cloud sensor presented in section II-A is placed on the side of the fuselage, slightly looking down as shown in Figure 5.

\section{B. Operation organization}

During a typical operation, five operators were working together each with a specific role:
- The atmospheric scientist is monitoring the real time sensor values collected by the UAVs. When he estimates that a UAV is crossing a cloud worth sampling, he requests to deploy a specific pattern to the mapmaker operator. He is also in charge of the global weather forecast to determine if clouds are coming within the next hour.

- The mapmaker operator is checking the real-time mapping process based on Gaussian Process Regression. Once he receives new instruction from the atmospheric scientist, he creates a new mission element with the desired parameters.

- The UAV operator is controlling the flights from the GCS. In particular, he is in charge of take-off, landing and waiting procedures, as well as the general safety of the flights. He is assisted by the safety pilot who is outside with the remote control and who is handling the planes.

- The flight director is the coordinator of the three other operators. He is checking the created mission and will decide if they should be accepted or rejected. He is also in charge of the coordination with the other teams sharing the airspace and is the point of contact for the local Air Traffic Control.

The general flight plan was to send the UAVs in a search mode first around eight kilometers from the coast to perform a hippodrome at an higher altitude than the cloud base as seen in Figure 6 (top). This altitude is extremely important and is determined from the previous flights of the day or based on the data from other teams of the EUREC4A campaign. In the case of a valid detection of a cloud, the operation sequence is launched by creating a new mission element and finally the UAVs operator will activate the mission mode to perform the required patterns. The video joined to the article is a replay of a real flight which presents the operation sequence.

\section{Sensor data filtering}

As seen in Figure 6 (bottom), the sensor data are noisy. A low-pass filter and a median filter have been added in order to give better detection results. Moreover, the detection of a border of a cloud is made thanks to a threshold combined with an hysteresis (red lines in the Figure 6 (bottom)). If the cloud sensor value goes above the higher red line, the UAV considers itself in the cloud; if it falls below the lower red line it considers itself out.

The flight patterns are currently dependent of the cloud sensor measurements as it is the best indication of the presence of clouds. Later, a combination with another sensor measurements will provide more accurate border detection. Meanwhile, the only way to validate the measurements is to compare visually the data with the on board videos recorded during the flight. Initial estimates of filter parameters and detection threshold are set according to the previous flight. When the UAV arrives at the desired altitude, the filter parameters are changed to reduce the noise of the signal and the detection thresholds are set manually based on the 

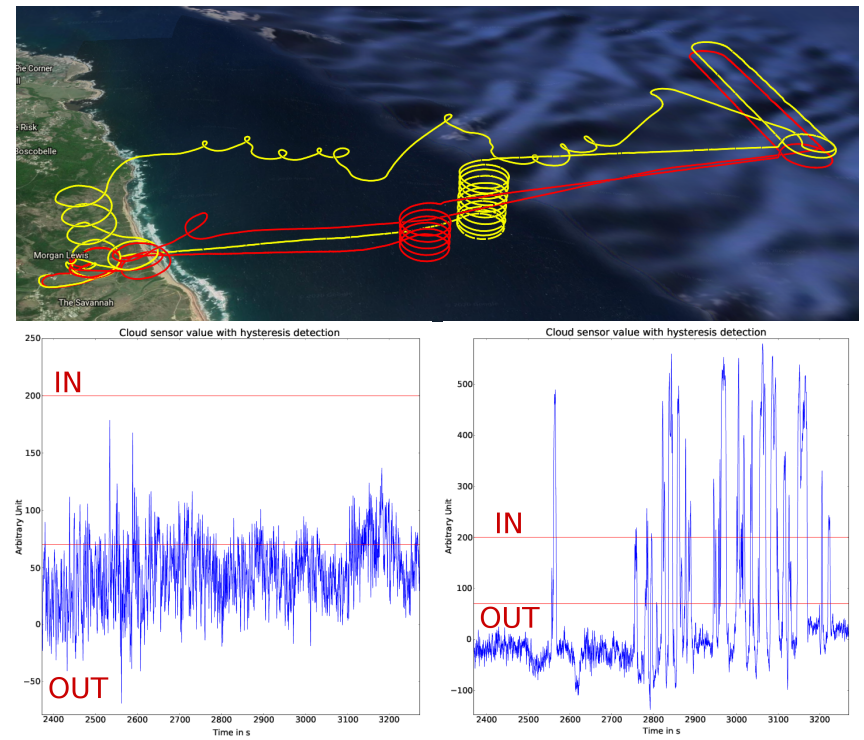

Fig. 6. Trajectories from a flight with 2 UAVs and the cloud sensor values (red trajectory corresponds to left plot and yellow trajectory to right plot). The horizontal lines are detection threshold with hysteresis, crossing upper line is entry detection, going back below bottom line is exit detection.

observation of the operator. Those parameters must also be modified according to the characteristics of the atmosphere. Several other constraints should be taken into account. In particular, the power consumed by the UAV directly impacts the cloud measurement. Moreover, the position of the sun (lower at the end of the day) disturbs the sensors with direct ray. The aerosol composition of the atmosphere also impacts the measurement but wasn't considered.

\section{Flight pattern trajectory analyzes}
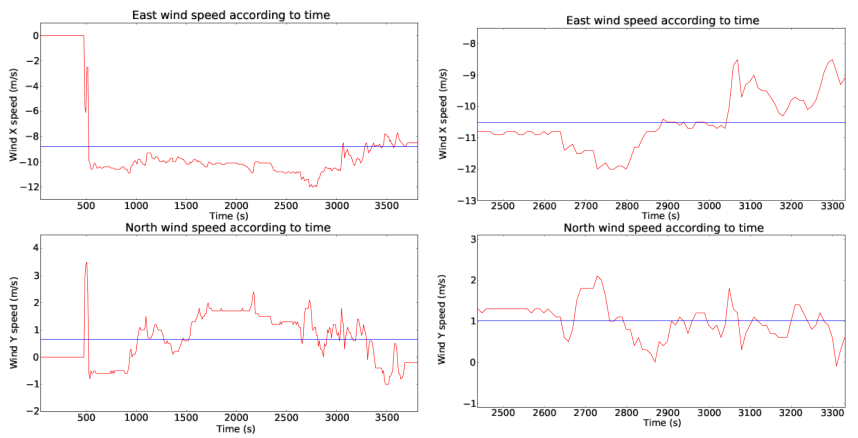

Fig. 7. Extracted relative wind during an entire flight at the left and a laps of time on the right

Figure 7 shows the wind estimated by the GCS based on the GPS track as presented in [11]. This method allows the computation of the airspeed when the UAV performs a turn with a constant speed. Thanks to this algorithm, the estimated value of the airspeed converges during a turn but diverges and looses accuracy in case of long straight lines. The particular location of the Barbados island guarantees a wind speed that is roughly constant in intensity and direction. However the algorithm takes time to converge after takeoff and is slightly increasing after reaching the cloud base. That is why only the part of the flight where the plane is actually chasing a cloud is considered. Moreover, considering the uncertainty on airspeed measurement and the low fluctuation of the real wind, it is preferable to consider an average speed rather than local values to correct the trajectory. In this case, the average speed of wind for the entire flight is about $9 \mathrm{~m} / \mathrm{s}$ from east whereas the average for the section is about $10.5 \mathrm{~m} / \mathrm{s}$.

Taking the first coordinates of the selected portion of the trajectory as a reference, the coordinates in the local cloud frame are computed by correcting the GPS points with the average wind speed vector previously estimated as follows:

$$
\begin{gathered}
\left(X_{i}\right)=\left(L_{o n g_{i}}-L_{\text {Long }}\right) \times R_{T} \times \cos \left(\text { Lat }_{i}\right) \\
\left(Y_{i}\right)=\left(\text { Lat }_{i}-L_{a t}\right) \times R_{T}
\end{gathered}
$$

where $L o n g_{0}$ and $L a t_{0}$ correspond to the longitude and latitude (in radian) of the origin point and $R_{T}$ is the radius of the earth in meters. By this end, the output values are the local positions in Earth frame. Then, the next operation is to subtract the relative wind to these values:

$$
\begin{gathered}
\left(X_{c}\right)=X_{i}-\left(\overline{W_{x}} \times\left(T_{i}-T_{0}\right)\right) \\
\left(Y_{c}\right)=Y_{i}-\left(\overline{W_{y}} \times\left(T_{i}-T_{0}\right)\right)
\end{gathered}
$$

where $X_{c}$ and $Y_{c}$ correspond to the local positions in cloud frame, $\overline{W_{x}}$ and $\overline{W_{y}}$ are the east and north components of the average wind speed and $T_{0}$ and $T_{i}$ are the time of reference and current point.
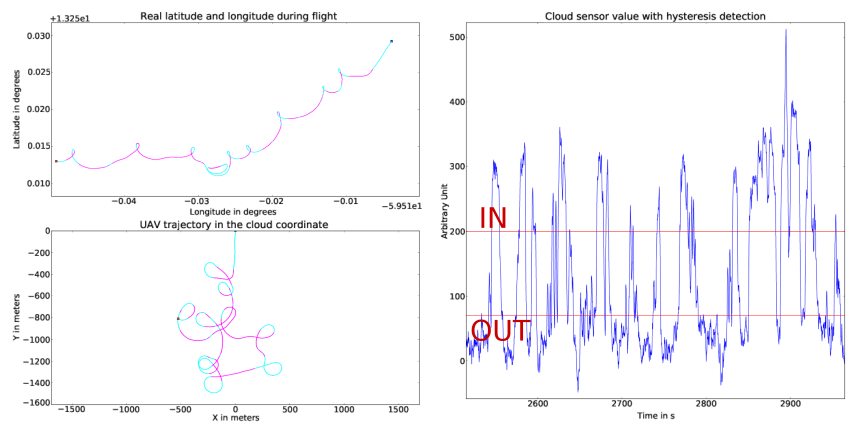

Fig. 8. Top left corner : real lat long plot of a flight section ; Bottom left corner: trajectory of the UAV in the cloud origin in meters (cyan/purple = outside/ inside); Right: Cloud sensor value getting during this section

Figure 8 shows a flight section during the execution of a Trinity flight pattern. The right plot is the cloud sensor value and the hysteresis chosen for this flight. The top left trajectory is the uncorrected one in Earth frame. The color shows when the plane is inside (purple) or outside the cloud (cyan). The bottom left curve is the corrected trajectory in local cloud frame. Now the shape of the Trinity is clearly visible as well as the location of the cloud in the center. The UAV is turning around the border as expected.

All the following figures are plotted in this corrected cloud frame.

The plots of Figure 9 show the resulting trajectories of a trinity pattern, which is more robust considering the small 

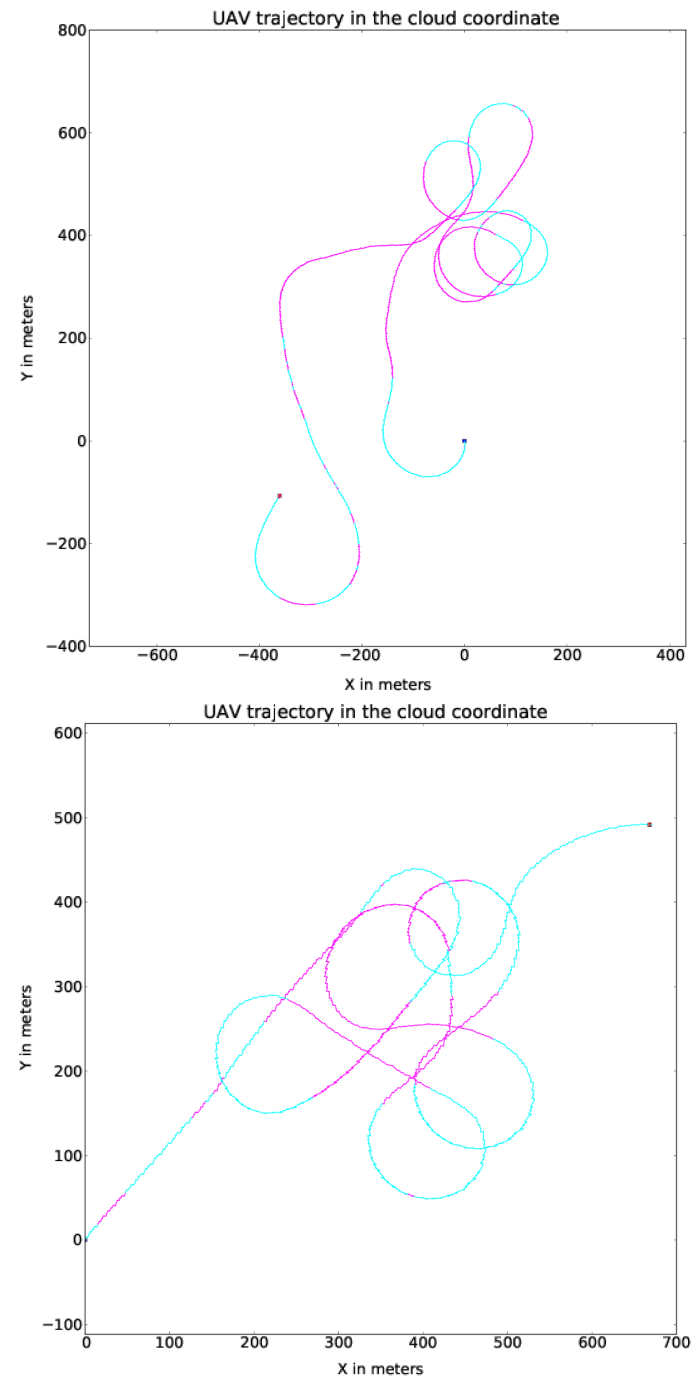

Fig. 9. Trajectories in the cloud coordinates resulting from the execution of a trinity pattern in two different cloud (cyan/purple = outside/inside)

size of the clouds. The turn around on each exit are all working. However, depending on the shape of the cloud and the limited airspace, they are not all able to make a complete turn around the clouds and can be trapped on one side (for instance in the top plot).

Figure 10 displays a trajectory of a UAV during a rosette pattern. The UAV crossed a first time the cloud, then it tried to do a turn around but was disturbed with several border detection which caused a delay. Once it went back in the cloud, it was guided by the update of the cloud center given by CAMS which result in a second straight line across the cloud. The second turn around is more significant of the pattern's expectation. This result is quite encouraging. However this pattern suffered from a lack of robustness in terms of cloud tracking and some adjustments are required to increase its efficiency.

Finally, Figure 11 shows the result of a lace pattern during a short laps of time. In the top part of the curve, the UAV was disturbed by a succession of border detection which results in a longer turn. During the flight campaign, the lace pattern

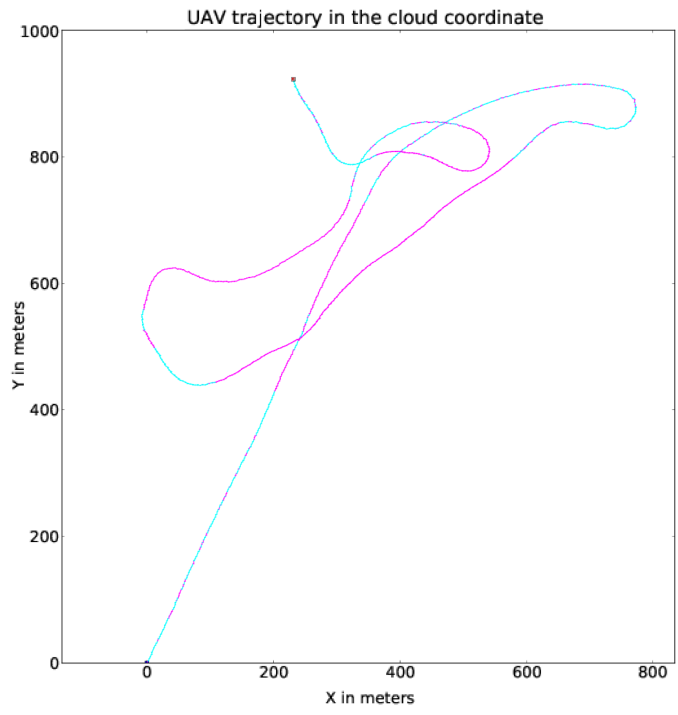

Fig. 10. Plot of the rosette pattern in the cloud coordinates (cyan/purple $=$ outside/inside)

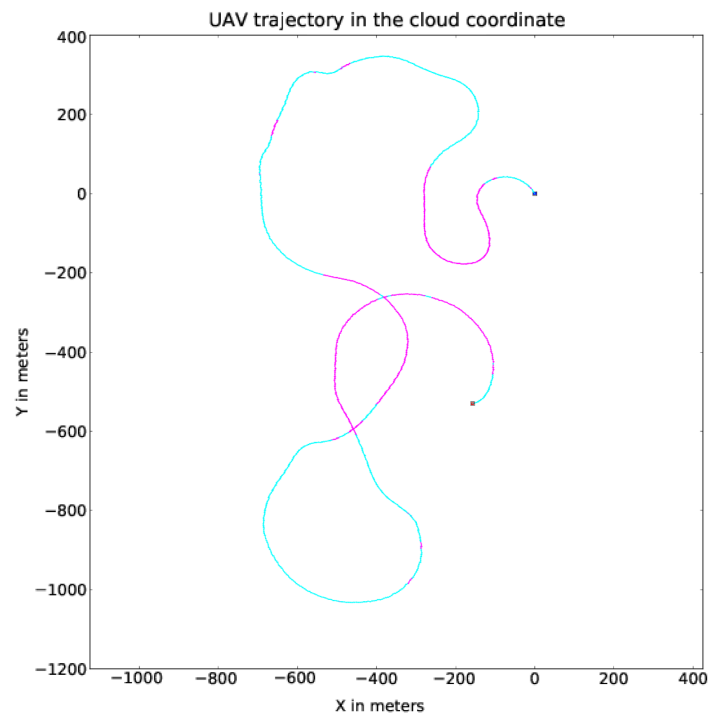

Fig. 11. Plot of the lace pattern in the cloud coordinates (cyan/purple $=$ outside/inside)

was not deployed a lot because of the size of the sampled cloud. In this case, the operator has decided to start this pattern because the estimated size was big enough to allow a tracking with it. Even if the track is really short and the cloud smaller than expected, the final behavior matches the expectations.

As the objective of the campaign was also to perform simultaneous sampling on the same cloud. Several multiUAV flights have been realized, but only one flight with two UAVs gave this opportunity. A first plane started the tracking and was joined by a second one already flying (Figure 12).

The use of several UAVs flying close to each other in a small airspace is highly demanding for the operators and 


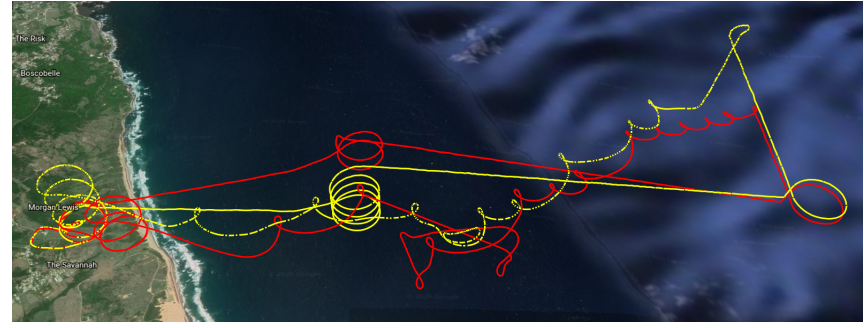

Fig. 12. KML trajectory of two UAVs during a double sampling

a loss of focus during a short time can quickly lead to a crash. To be safely operated, it is necessary to develop a control and a plan manager for the fleet. This will add more self-sufficiency on the UAVs, giving more time to the UAV operator.

TABLE I

SUMMARY TABLE OF THE FLIGHTS DURING THE EXPERIMENTAL BARBADOS FLIGHT CAMPAIGN

\begin{tabular}{|c||c|l|}
\hline $\begin{array}{c}\text { Number of } \\
\text { flights }\end{array}$ & 48 & 22 flights realized with two UAVs \\
\hline Data recorded & $\begin{array}{c}45 \\
\text { hours }\end{array}$ & $\begin{array}{l}\text { Flight time average around } 53 \text { minutes } \\
\text { per flight }\end{array}$ \\
\hline $\begin{array}{c}\text { Calibration } \\
\text { flights }\end{array}$ & 23 & $\begin{array}{l}\text { Cloud sensor and UAV calibration, vali- } \\
\text { dation of the flight pattern }\end{array}$ \\
\hline $\begin{array}{c}\text { Measurement } \\
\text { flights }\end{array}$ & 25 & $\begin{array}{l}\text { Vertical profile and cloud tracking mis- } \\
\text { sion }\end{array}$ \\
\hline $\begin{array}{c}\text { Usable flights } \\
\text { Tracking of a cloud during more than } 2 \\
\text { minutes. Average following time around } \\
5 \text { minutes per tracking. }\end{array}$ \\
\hline
\end{tabular}

Table I presents a summary of the flights during the experimental flight campaign. To conclude, this first campaign was intense and allowed to gather data as well as highlighted possible future improvements.

\section{CONCLUSION AND FUTURE WORK}

The adaptive flight pattern algorithms have been developed to satisfy a particular need of collecting data inside clouds. The evaluation of the performances of this approach is not something easy to measure. Moreover, deploying several UAVs in the proper meteorological conditions is particularly challenging. It requires an important preparation with simulations and flight tests, and a complex logistic for the actual field campaign.

The Barbados campaign offered the possibility to test the flight patterns in real conditions, with encouraging results from the UAV fleet control perspective. The pattern that was mostly executed during the campaign was the Trinity, so the measurements collected are mainly along the cloud borders. From the atmospheric research side, these data are an asset to better understand the interaction of cumulus clouds with their environment.

The observations made during the campaign on the flight patterns, the wind estimation, the fleet control and the sensors measurements will play a key role to improve the system. Ongoing work is focusing on the flight patterns monitoring to improve their robustness and to allow to recover faults, e.g. when losing track of the cloud. Vision sensors and algorithms are planned to be added to the system to have a better estimate of the initial position and shape of the clouds. Finally, future researches should also focus on the development of a new control and decision algorithm of the fleet to lighten the operator's workload and to deploy a larger fleet.

\section{ACKNOWLEDGMENTS}

The authors would like to thank all the engineers and researchers from ENAC, LAAS-CNRS and Météo-France involved in the NEPHELAE project and the other teams of the EUREC4A campaign.

The NEPHELAE project is funded by the French National Research Agency (ANR). This work is funded by the Université de Toulouse and Région Occitanie.

\section{REFERENCES}

[1] A. P. Siebesma et al. Shallow Cumulus Convection, pp. 441-486. Springer Netherlands, Dordrecht, 1998.

[2] B. D. Reineman et al. The use of ship-launched fixed-wing UAVs for measuring the marine atmospheric boundary layer and ocean surface processes. Journal of Atmospheric and Oceanic Technology, 33(9):2029-2052, 2016.

[3] T. Egorova et al. Estimation of gaseous plume concentration with an unmanned aerial vehicle. Journal of Guidance, Control, and Dynamics, 39(6):1314-1324, 2016.

[4] R. G. Harrison and K. A. Nicoll. Note: Active optical detection of cloud from a balloon platform. Review of Scientific Instruments, 85(6):066104, 2014.

[5] T. Verdu et al. Flight patterns for clouds exploration with a fleet of uavs. In 2019 International Conference on Unmanned Aircraft Systems (ICUAS), pp. 231-237, June 2019.

[6] G. Hattenberger et al. Using the Paparazzi UAV System for Scientific Research. In IMAV 2014, International Micro Air Vehicle Conference and Competition 2014, pp. pp 247-252, Delft, Netherlands, August 2014.

[7] C. Reymann et al. Adaptive sampling of cumulus clouds with UAVs. Autonomous Robots, 42(2):491-512, 2018.

[8] N. Maury et al. Adaptative cloud exploration by uav fleet in simulated cumulus fields for studying entrainment and microphysics of clouds. In AGU Fall Meeting 2019, 2019.

[9] C. Lac et al. Overview of the Meso-NH model version 5.4 and its applications. Geoscientific Model Development, 11(5):1929-1969, May 2018. Publisher: Copernicus GmbH.

[10] S. Bony et al. EUREC4A: A Field Campaign to Elucidate the Couplings Between Clouds, Convection and Circulation. Surveys in Geophysics, 38(6):1529-1568, November 2017.

[11] S. Mayer et al. A "no-flow-sensor" wind estimation algorithm for unmanned aerial systems. International Journal of Micro Air Vehicles, 4(1):pp 15-30, March 2012. 